\title{
Underlying mechanisms of the effect of minocycline against Candida albicans biofilms
}

\author{
$\mathrm{LI} \mathrm{ZOU}^{1 *}, \mathrm{ZHAO} \mathrm{MEI}^{2,3^{*}}$, TAO GUAN ${ }^{1}, \mathrm{BO}_{\mathrm{ZHANG}}{ }^{1}$ and $\mathrm{QUN} \mathrm{DENG}^{1}$ \\ Departments of ${ }^{1}$ Clinical Laboratory and ${ }^{2}$ Pharmacy, The People's Hospital of China Three Gorges University; \\ ${ }^{3}$ Medical College of China Three Gorges University, Yichang, Hubei 443000, P.R. China
}

Received April 20, 2020; Accepted October 26, 2020

DOI: $10.3892 /$ etm.2021.9857

\begin{abstract}
Minocycline (MH) is a broad-spectrum antimicrobial agent and semisynthetic tetracycline derivative, which has been widely used in the clinic due to its efficacy. Having the strongest anti-microbial effect, $\mathrm{MH}$ exceeded the traditional scope of antibiotics and its previously unknown antifungal activity is also gradually being discovered. To preliminarily investigate the inhibitory effect of $\mathrm{MH}$ on Candida albicans (C. albicans), changes of cell growth, hyphal formation and transition, biofilm production and signaling pathway gene expression of $C$.albicans in the presence of $\mathrm{MH}$ were assessed in the present study. An XTT reduction assay was performed to quantitatively detect the metabolic activity of biofilms and evaluate the inhibition of MH on this. The results suggested that biofilm formation was clearly inhibited by $67 \%(\mathrm{P}<0.0001)$ in the presence of $250 \mu \mathrm{g} / \mathrm{ml} \mathrm{MH}$, while mature biofilms were not significantly affected. In addition, $\mathrm{MH}$ inhibited the transition from yeast to hypha in a dose-dependent manner. Furthermore, reverse transcription-quantitative PCR revealed that several hyphae- and adhesion-specific genes associated with the Ras/cyclic (c)AMP/protein kinase A (PKA) pathway were differentially expressed following $\mathrm{MH}$ treatment, including downregulation of ras family GTPase (RAS1), adenylyl cyclase-associated protein 1 (CAPl), thiamin pyrophosphokinase 1 (TPK1), adenylate cyclase (CDC35), transcription factor (TEC1), agglutinin-like protein 3 (ALS3) and hyphal wall protein $1(H W P 1)$ and upregulation of $E F G 1$ (enhanced filamentous growth protein 1 gene) and $P D E 2$
\end{abstract}

Correspondence to: Dr Qun Deng, Department of Clinical Laboratory, The People's Hospital of China Three Gorges University, 2 Jiefang Road, Yichang, Hubei 443000, P.R. China

E-mail: dengqun77@163.com

*Contributed equally

Abbreviations: $\mathrm{MH}$, minocycline; TE, tetracycline; DO, doxycycline; TGC, tigecycline; RT-qPCR, reverse transcription-quantitative PCR; SDA, Sabouraud dextrose agar

Key words: minocycline, Candida albicans, morphology, anti-biofilm, antifungal activity (high-affinity phosphodiesterase gene). The most obviously changed genes were TPK1,HWP1 and RASl, downregulated by 0.33 -, 0.48 - and 0.55 -fold, respectively. It was suggested that $\mathrm{MH}$ is associated with alterations in the morphology of $C$. albicans, such as the repression of hypha and biofilm formation of cells, and MH affected the Ras/cAMP pathway to regulate the expression of cAMP-associated genes.

\section{Introduction}

Candida albicans (C. albicans) is considered the most common opportunistic fungal pathogen in humans due to its widespread distribution in the environment. It has a high propensity to cause invasive infections on mucosal surfaces and is particularly life-threatening in individuals with immunodeficiency and dysbacteriosis, as systemic candidiasis has a high mortality rate in such patients $(1,2)$. Fungi continue to vary and mutate in response to environmental and body stimuli and the antifungal drugs available are simply insufficient for the clinical/pharmacological treatment of patients. In addition, the incidence of $C$. albicans resistance increased rapidly due to the widespread and long-term usage of limited antifungal drugs, causing additional difficulty in treatment and ultimately leading to refractory fungal infections (3-5).

One of the morphological characteristics that distinguish C. albicans from other organisms is polymorphism and pleomorphism. C. albicans may have three distinct phenotypic characteristics: A yeast-like form at $25^{\circ} \mathrm{C}$, and additional pseudohyphae and hyphae forms at $37^{\circ} \mathrm{C}$, which are commonly referred to as filamentous morphologies $(6,7)$. All three forms may coexist and transform with the change of environmental stimuli. C. albicans biofilms, as an important barrier, are considered significant for pathogenicity and virulence, as well as adhesion and infectivity, which are contributing factors in the process of infecting the host and are able to withstand the stimulation of the external environment and drugs. Therefore, numerous clinical antifungal drugs are mainly resistant to biofilms. The major conventional antifungal drugs currently in clinical use are fluconazole and amphotericin B. Studies have demonstrated that the frequent and excessive use of antifungal drugs led to the notorious resistance of $C$. albicans to a wide variety of clinical antifungal drugs, including the aforementioned two $(8,9)$.

The contradiction between effective antifungal therapy and finite available clinical antifungal drugs is increasingly 
prominent. Therefore, novel antifungal agents are urgently required in order overcome the resistance of $C$. albicans biofilms. Recent scientific studies have re-evaluated tetracycline (TE) derivatives, such as minocycline (MH), TE, doxycycline (DO), tigecycline (TGC), oxytetracycline and demethylchlortetracycline, for their potential antifungal activity as well as old traditional antibiotics combined with antifungal drugs to achieve an outstanding effect against fungal infections (10-13).

$\mathrm{MH}$, a TE derivative, is a traditional antimicrobial agent that inhibits bacterial protein synthesis, which is extensively used in the clinic due to its large spectrum of antibiotic activity. MH has a strong effect on gram-positive bacteria, including Staphylococcus aureus, with the effect on gram-negative bacilli being slightly weaker. When MH is combined with other antifungal agents, such as fluconazole, the antifungal effect of combination treatment is significantly stronger than that of MH used alone $(10,11,14,15)$. Previous studies have reported that $\mathrm{MH}$ is able to mediate several biological phenomena and is associated with the capacity of morphological transformation from yeast to hypha in C. albicans (14).

The RAS/cyclic (c)AMP/protein kinase A (PKA) signaling pathway has an impact on cell growth, differentiation, protein secretion and transport. Hogan and Sundstrom (16) and Phillips and Crowe (17) determined separately that in C. albicans, the Ras signaling pathway mediates the morphological transition and biofilm formation, and contains certain vital hypha-specific genes, including ras family GTPase $(R A S 1)$, thiamin pyrophosphokinase 1 (TPK1), enhanced filamentous growth protein 1 (EFG1), TECl, CAP1 (adenylyl cyclase-associated protein 1), phosphodiesterase 2 (PDE2) and adenylate cyclase (CDC35), as well as certain adhesion-specific genes, including agglutinin-like protein $3(A L S 3)$ and hyphal wall protein $1(\mathrm{HWPl})$. Based on the results of these studies, MH may be a promising and effective antifungal agent. It is able to alleviate the urgent problem of drug resistance and short supply of antifungal drugs, which may provide a major breakthrough in the clinical treatment of fungal infections. However, in order to understand the antifungal activity of $\mathrm{MH}$ thorough and systematic evaluation is required and the effect of $\mathrm{MH}$ on C.albicans biofilms and the mechanisms underlying this require further investigation. In the present study, it was hypothesized that the antifungal effect of $\mathrm{MH}$ on C. albicans is mainly associated with biofilm formation and its regulation is closely associated with the RAS/cAMP/PKA pathway. In the present study, the in vitro antifungal activity of the widely used antimicrobial $\mathrm{MH}$ in C. albicans was assessed, and whether its potential antifungal mechanism is associated with the Ras/cAMP signaling pathway was systematically explored to verify the validity of this hypothesis.

\section{Materials and methods}

Strains, antibacterial agents and growth conditions. A total of four TE derivatives were examined in the present study: MH, TE, DO and TGC. The TH drug susceptibility test (TE, $30 \mu \mathrm{g}$; DO, $30 \mu \mathrm{g}$; TGC, $15 \mu \mathrm{g}$ ) was obtained from BioMérieux and $\mathrm{MH}$ drug papers $(30 \mu \mathrm{g})$ from Merck KGaA. MH was dissolved in DMSO. A high concentration of the original solution was prepared, stored at $-20^{\circ} \mathrm{C}$ away from light and then diluted to the desired final concentration prior to use. The final content of DMSO was $<2 \%$ and had no effect on cells in the present study.

The reference strain C. albicans ATCC 90028 was a gift obtained from Renmin Hospital of Wuhan University. All clinically isolated yeast strains used in the present study were isolated at the People's Hospital of China Three Gorges University (Yichang, China) and stored in the central laboratory. All strains used in the present study were identified according to the standard morphological criteria (18). Strains were routinely cultured on Sabouraud dextrose agar (SDA) plates at $28^{\circ} \mathrm{C}$ for $24-48 \mathrm{~h}$, then streaked out onto Yeast Extract-Peptone-Dextrose (YPD) liquid medium and grown at $30^{\circ} \mathrm{C}$ in a shaking incubator for $24-48 \mathrm{~h}$ until they reached the logarithmic growth phase and the absorbance was measured at $600 \mathrm{~nm}$. Hyphal inductions were performed at $37^{\circ} \mathrm{C}$ in liquid medium, and Spider and RPMI-1640 medium were prepared as previously described (19).

Effect of TE derivatives on the growth of yeast strains (Kirby-Bauer disc diffusion method). All strains were adjusted to $0.5 \mathrm{McF}$ arland bacterial suspensions and then evenly spread to a Mueller-Hinton agar plate (Thermo Fisher Scientific, Inc.). Once the surface of the plate was dry, sensitivity test papers containing one of the $4 \mathrm{TE}$ antibiotics were applied. All plates were incubated for $24 \mathrm{~h}$ at $28^{\circ} \mathrm{C}$ and the diameter of the inhibition ring was measured.

Effect of MH on planktonic cells in C. albicans. Antifungal susceptibility testing was performed in 96-well tissue culture plates (Corning, Inc.) according to the protocol of the Clinical and Laboratory Standards Institute (20). In brief, C. albicans ATCC 90028 was prepared with initial cells at $1 \times 10^{5} \mathrm{CFU} / \mathrm{ml}$ and cultured in YPD liquid medium for $48 \mathrm{~h}$ at $37^{\circ} \mathrm{C}$. To evaluate the effect of $\mathrm{MH}$ on the cell growth in C.albicans ATCC 90028, an inoculum concentration of yeast cells at $\sim 1 \times 10^{6}$ cells $/ \mathrm{ml}$ was added into liquid RPMI 1640 medium containing $\mathrm{MH}$ at concentrations of $0,125,250,500$ and $1,000 \mu \mathrm{g} / \mathrm{ml}$, incubated in $96-$ well tissue culture plates at $37^{\circ} \mathrm{C}$ and shaken at $200 \mathrm{rpm} .(2.2 \mathrm{x} \mathrm{g})$. The cells were monitored every $4 \mathrm{~h}$ and counted at the predetermined time-points $(0,4,8,12,16,20,24,28$ and 32 h) using a Multiskan GO Microplate reader (Thermo Fisher Scientific, Inc.) to measure the absorption/optical density (OD) at $600 \mathrm{~nm}$, and the background optical densities were already subtracted. A total of three independent experiments were performed (21).

Hyphal formation assessment. To further estimate whether the MH affects bud-to-hypha transition, a fixed amount of C.albicans cells $\left(1 \times 10^{6}\right.$ cells $\left./ \mathrm{ml}\right)$ was cultured in several nutritious hypha transition media. The spider and RPMI 1640 media (Sangon Biotech Co., Ltd.) were supplemented with $20 \%$ fetal bovine serum (FBS; Cytiva) in 24-well tissue culture plates containing various concentrations of $\mathrm{MH}$ and incubated at $37^{\circ} \mathrm{C}$ for $3 \mathrm{~h}$, and hyphal formation was evaluated microscopically to count 200 cells and calculate the percentage of hyphal formation, as previously described (14).

Antifungal activity against mature biofilms. The $C$. albicans cells in the logarithmic growth phase were collected and 
centrifuged, RPMI 1640 medium was added to adjust the concentration to $1 \times 10^{6} \mathrm{CFU} / \mathrm{ml}$ and $100 \mu \mathrm{l}$ bacterial liquid was added to the 96 -well culture plate for $90 \mathrm{~min}$ of adhesion at $37^{\circ} \mathrm{C}$. The medium was gently sucked off with a sample gun, non-adherent planktonic cells were removed, $100 \mu \mathrm{l}$ fresh RPMI 1640 medium was added and the plate was incubated at $37^{\circ} \mathrm{C}$ for $24 \mathrm{~h}$ until the mature biofilm was formed (20). The biofilm supernatant was then aspirated, RPMI 1640 with MH was added to for a 24-h incubation, the growth of the biofilm was observed under a microscope and an XTT kit (Sangon Biotech Co., Ltd.) was used to evaluate the formed mature biofilm. Similarly, in order to detect the effect of MH on the formation of biofilms, MH was added to fresh RPMI 1640 after 90 min of adhesion; subsequent methods were described above until biofilm formation to observe the anti-biofilm effect of $\mathrm{MH}(20,22)$.

Gene expression analysis of $C$. albicans-specific genes. Following $\mathrm{MH}$ treatment overnight, $C$. albicans cells were washed and collected, and a Fungal RNA out kit (Sangon Biotech Co, Ltd.) was used to extract and isolate the total RNA of $C$. albicans according to the manufacturer's protocol and the standard method. The OD was measured at 260-280 nm using a Nanodrop 2000 Ultraviolet spectrophotometer (Thermo Fisher Scientific, Inc.) to test the nucleic acid concentration and purity of the RNA. The integrity of the RNA was assessed using denatured electrophoresis through a $1 \%$ agarose-3-(N-morpholino) propanesulfonic acid gel (Sangon Biotech Co., Ltd.). A reverse transcription kit (Takara Bio, Inc.) was used to reverse-transcribe single-stranded RNA $(1 \mu \mathrm{g})$ into cDNA. Next, reverse transcription-quantitative PCR (RT-qPCR) was performed using a 7500 real-time PCR system (Thermo Fisher Scientific, Inc.); hyphal-specific and biofilm-associated genes of $C$. albicans, as well as their transcriptional regulators, were analyzed in the present study, and the primers are listed in Table I. The associated genes were as follows: RAS1, a major transcription factor involved in the MAPK pathway; EFG1, transcription factor in the cAMP/PKA pathway; GAP1, which encodes a general amino acid permease; ALS3 and HWP1, which encode agglutinin-like protein 3 and hyphal wall protein 1, respectively, and are activated by EFG1; CDC35 (coding for adenylyl cyclase); TPK1 (PKA catalytic subunits); PDE2 (high-affinity phosphodiesterase gene); TEC1, a transcription factor; ACT1, a housekeeping gene that has the ability to express stably and consistently in organisms, and was chosen as an endogenous control gene for correcting the loading inaccuracies (23). The final values were calculated by the $2^{-\triangle \Delta C q}$ method (24).

The PCR conditions were programmed and divided into three steps according to the experimental conditions and purposes. The first step was initial denaturation at $95^{\circ} \mathrm{C}$ for 5 min, where double-stranded DNA templates break hydrogen bonds under thermal action to form single-stranded DNA. The second step was annealing at $55-65^{\circ} \mathrm{C}$, with the continuous gradient temperature set at a heating rate of $0.1^{\circ} \mathrm{C} / \mathrm{sec}$ to match the appropriate suitable solution curve temperature, the system temperature was lowered and the primer bound to the DNA template to form a local double strand. This step eventually went through 40 cycles of amplification and quantification at $95^{\circ} \mathrm{C}$ for $10 \mathrm{sec}, 55^{\circ} \mathrm{C}$ for $30 \mathrm{sec}$ and $72^{\circ} \mathrm{C}$ for $15 \mathrm{sec}$ with a
Table I. PCR primers used for detection of gene expression in Candida albicans.

\begin{tabular}{ll}
\hline Primer & \multicolumn{1}{c}{ Sequence (5'-3') } \\
\hline CAP1-F & GAACCACCATCAACATCA \\
CAP1-R & CAACCCATCAATATCAAGT \\
TPK1-F & AGAAGTTCAAGATGTGACTTAT \\
TPK1-R & CATCATCAGAACCACCTTGT \\
CDC35-F & TCCATGTCAAATATGCCAACG \\
CDC35-R & CTTCACATCCCAACTTTCAGG \\
EFG1-F & TCCATGTCAAATATGCCAACG \\
EFG1-R & TGGATTCATACCGTATTGGTCATTA \\
TEC1-F & TGGATTCATACCGTATTGGTCATTA \\
TECI-R & TCGGGCAATCCTTTGAATAAA \\
RAS1-F & GTGGTGGTGTTGGTAAATC \\
RAS1-R & TTCTTGTCCAGCAGTATCT \\
PDE2-F & TGCTGTGGGACATTGGAG \\
PDE2-R & GGCGGAAATTATGGAACG \\
ALS3-F & GTGATGCTGGATCTAACGGTATTG \\
ALS3-R & GTCTTAGTTTTGTCGCGGTTAGG \\
HWP1-F & CGGAATCTAGTGCTGTCGTCTCT \\
HWP1-R & CGACACTTGAGTAATTGGCAGATG \\
ACT 1-F & TTGATTTGGCTGGTAGAG \\
ACT 1-R & ATGGCAGAAGATTGAGA \\
\hline
\end{tabular}

F, forward primer; R, reverse primer; CAP1, adenylate cyclase-associated protein 1; TPK1, thiamin pyrophosphokinase 1; CDC35, adenylate cyclase; EFG1, enhanced filamentous growth protein 1; Tec1, transcription factor; RAS1, ras family GTPase; PDE, 2,3',5'-cyclic-nucleotide phosphodiesterase; ALS3, agglutinin-like protein 3; HWP1, hyphal wall protein 1; ACT 1, actin related gene 1 .

continuous single fluorescence measurement and end. The last step was a final extension maintained at $72^{\circ} \mathrm{C}$ for $5-10 \mathrm{~min}$. dNTP was used as raw material and primers started from the 3 'end, extending from the 5' to 3 ' end, to synthesize DNA strands complementary to the template, followed by a cooling step at $42^{\circ} \mathrm{C}$.

Statistical analysis. Values are expressed as the mean fold change \pm standard deviation of three independent experiments. Statistical analyses were performed using GraphPad Prism 5.0 (GraphPad Software, Inc.). One-way analysis of variance with Tukey's post hoc test was used to evaluate the statistical significance of differences among the treatment groups. $\mathrm{P}<0.05$ were considered to indicate a statistically significant difference.

\section{Results}

Inhibitory effects of TE derivatives on yeasts. The results of the drug sensitivity test are presented in Fig. 1. MH had the most obvious inhibition circle among the four TE derivatives (ME, TE, DO and TGC). By measurement and quantification, the ratio of the inhibition zone diameter $\geq 14 \mathrm{~mm}$ for $\mathrm{MH}$ on C. albicans was determined to be $80 \%$, that on C.tropicalis was $96 \%$ and that on $C$. Portuguese was $87 \%$. TE, DO and 


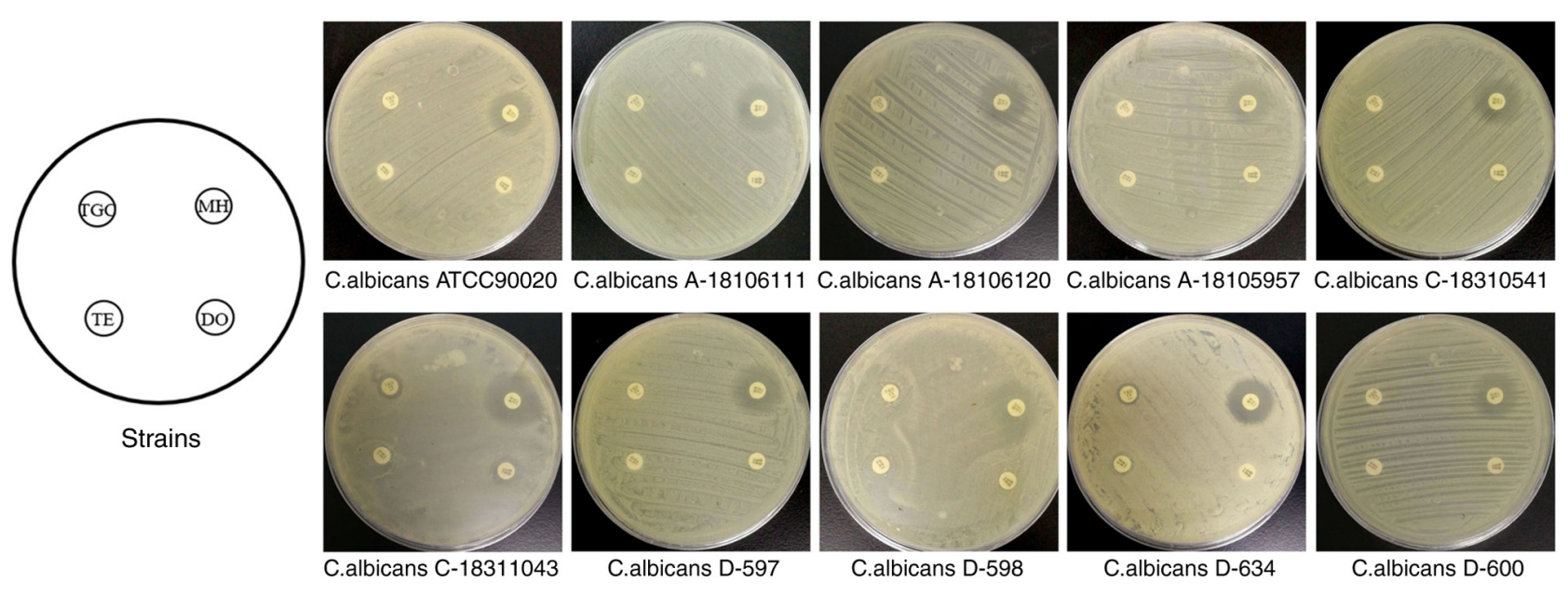

Figure 1. Antifungal effects of 4 TE derivatives. $\mathrm{MH}$ had a marked antifungal activity against $C$. albicans and DO had a slight antifungal activity against C. albicans, while TE and TGC had no antifungal activity. TE, tetracycline; MH, minocycline; DO, doxycycline; C. albicans, Candida albicans; TGC, tigecycline.

TGC exhibited no sufficient antifungal activity against C. albicans with the inhibition zone diameter being $6-10 \mathrm{~mm}$, $<14 \mathrm{~mm}$ in all strains.

Effects of $\mathrm{MH}$ on C. albicans growth. In the presence of $\mathrm{MH}$ at the concentrations of $125,250,500$ and $1,000 \mu \mathrm{g} / \mathrm{ml}$, a significant growth inhibition of $C$. albicans was observed in the treatment group as compared to the control group without MH treatment (Fig. 2). At $250 \mu \mathrm{g} / \mathrm{ml}$, MH exhibited the most potent inhibitory activity on $C$. albicans growth among all concentrations used. After $8 \mathrm{~h}$ of incubation in liquid culture, the growth curves of $C$. albicans began to diverge and exhibit visible differences. After $32 \mathrm{~h}$, the growth was significantly restricted in RPMI-1640 medium with $\mathrm{MH}$ at $125 \mu \mathrm{g} / \mathrm{ml}$, leading to a $59.9 \%$ reduction $(\mathrm{P}<0.01)$, and in the presence $250 \mu \mathrm{g} / \mathrm{ml} \mathrm{MH}$, the maximum inhibition was $68.0 \%(\mathrm{P}<0.01)$ as compared to the control group. However, with further increases in the concentration of $\mathrm{MH}$, the growth inhibition of planktonic C.albicans decreased.

MH regulates the hyphal formation process of $C$. albicans. Morphological changes were observed under light microscopy following incubation for $4 \mathrm{~h}$ and the quantitative results suggested that MH inhibited hyphal growth induction at all tested concentrations. More specifically, the maximum hypha formation inhibition occurred at the lowest concentration of MH $(125 \mu \mathrm{g} / \mathrm{ml})$ in both RPMI-1640 and Spider media and the degree of hypha inhibition slightly decreased with the increase of the $\mathrm{MH}$ concentration (Fig. 3A). The hypha rate of $\sim 200$ cells for each sample was determined using light microscopy (Fig. 3B). In drug-free medium, $>70 \%$ of $C$. albicans cells converted to the hyphal morphology after $4 \mathrm{~h}$ of incubation, with only a small percentage retaining their yeast form. However, following incubation with 125 and $250 \mu \mathrm{g} / \mathrm{ml} \mathrm{MH}$, the hypha formation rate was only $11.75 \pm 2.86 \%$ with a significant inhibition of hyphae in all media $(\mathrm{P}<0.001)$. Following incubation with $500 \mu \mathrm{g} / \mathrm{ml} \mathrm{MH}$, the hypha rates were $46.00 \pm 5.29 \%$ in RPMI-1640 medium and 23.00 $\pm 2.64 \%$ in Spider medium, both were significantly different from the control group $(\mathrm{P}<0.001)$. Following incubation with $1,000 \mu \mathrm{g} / \mathrm{ml} \mathrm{MH}$, the

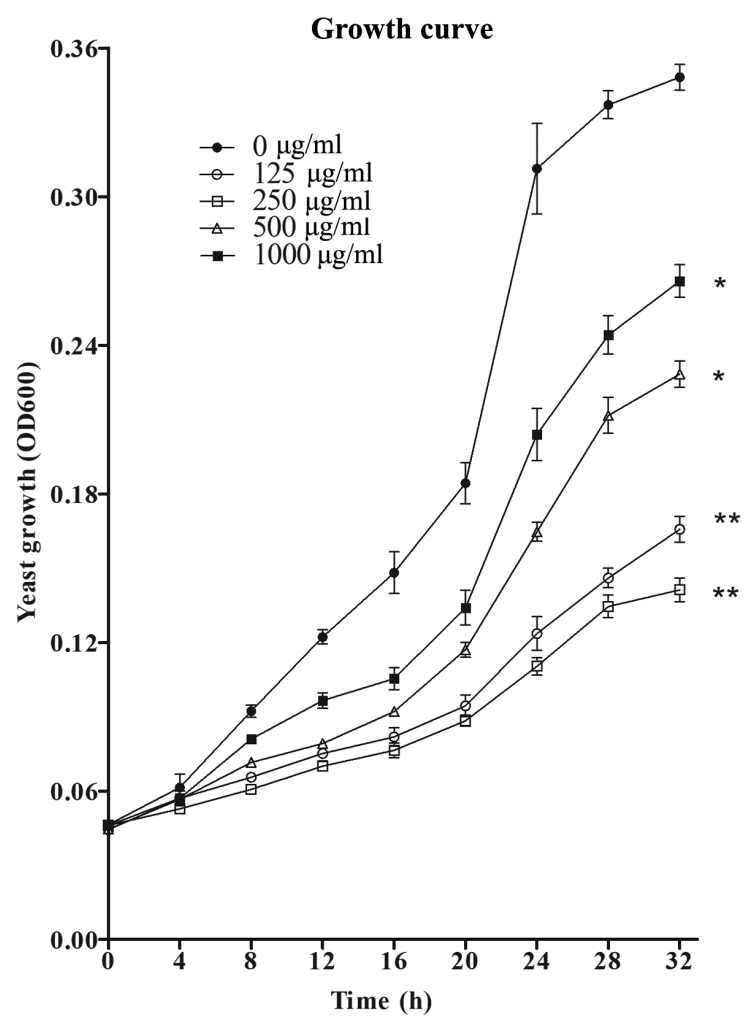

Figure 2. Effects of different concentrations of $\mathrm{MH}$ on fungal cell growth of the Candida albicans strain ATCC 90028. ${ }^{*} \mathrm{P}<0.05 ;{ }^{* * *} \mathrm{P}<0.01$. OD, optical density; $\mathrm{MH}$, minocycline.

hypha rates were $54.33 \pm 4.04 \%$ in RPMI-1640 medium and $35.33 \pm 2.51 \%$ in Spider medium, both were significantly different from the control group $(\mathrm{P}<0.01)$.

MH inhibits C.albicans biofilms. An XTT reduction assay was performed to examine the potential of $\mathrm{MH}$ to inhibit biofilm formation in vitro. As presented in Fig. 4, the biofilms formed by $C$. albicans decreased in an $\mathrm{MH}$ dose-dependent manner in terms of their density and thickness. More specifically, $\mathrm{MH}$ inhibited biofilm formation by $52 \%$ at $125 \mu \mathrm{g} / \mathrm{ml}(\mathrm{P}<0.001$ 
A

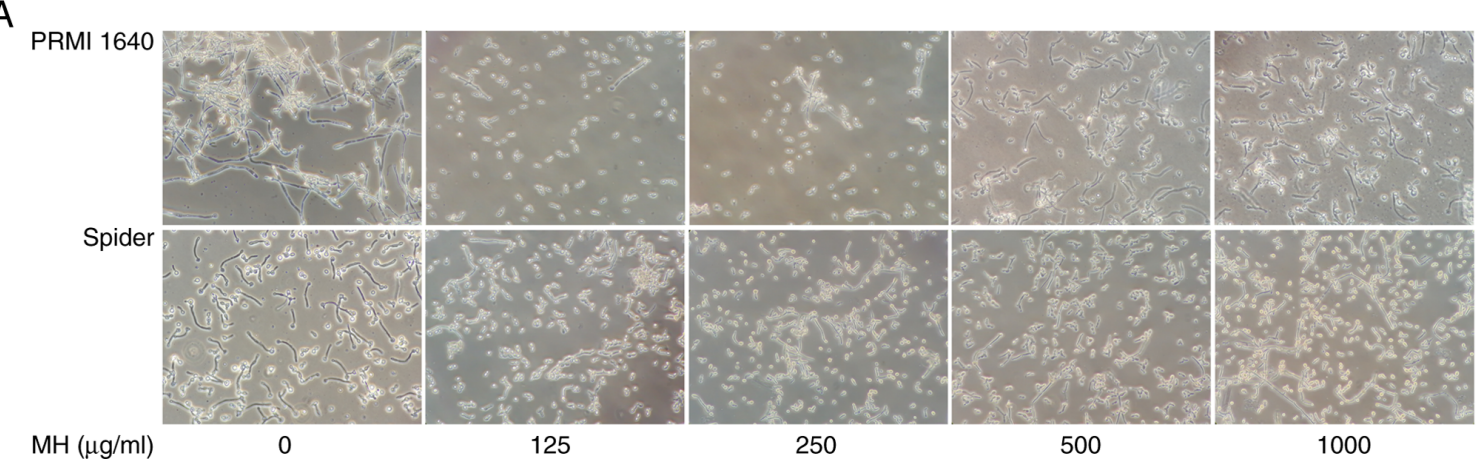

B

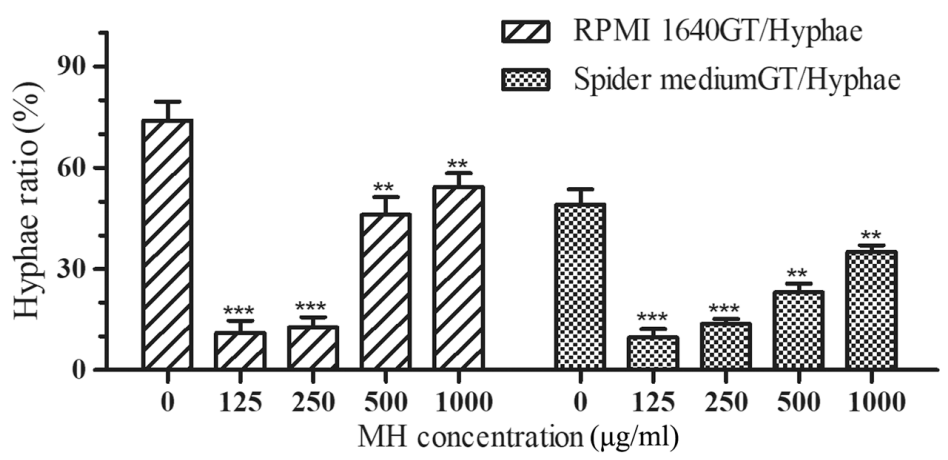

Figure 3. (A) Effects of MH on hyphal formation in C. albicans. Cells (1 $1 \times 10^{6}$ cells $\left./ \mathrm{ml}\right)$ were grown in Spider or RPMI 1640 media supplemented with $20 \%$ FBS. Photomicrographs of C. albicans ATCC 90028 grown at $37^{\circ} \mathrm{C}$ for $4 \mathrm{~h}$. MH had an obvious trend to inhibit filamentous growth as compared with cells incubated in drug-free medium (magnification, $\mathrm{x} 400$ ). (B) The hyphae rate was counted in a minimum of 200 cells from each sample following $4 \mathrm{~h}$ of cultivation. The results indicated that $\mathrm{MH}$ was able to inhibit hyphae formation at all concentrations, with the effects of $125 \mathrm{and} 250 \mu \mathrm{g} / \mathrm{ml} \mathrm{MH}$ being most significant. ${ }^{* *} \mathrm{P}<0.01$; ${ }^{* * * *} \mathrm{P}<0.001$ vs. control. $\mathrm{MH}$, minocycline; C. albicans, Candida albicans; FBS, fetal bovine serum.

A

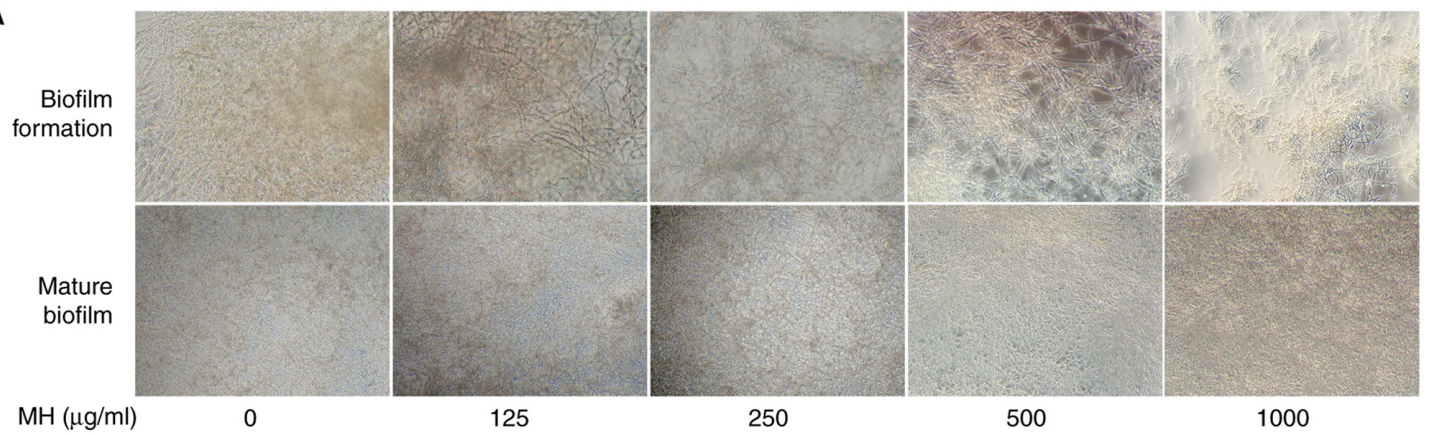

B

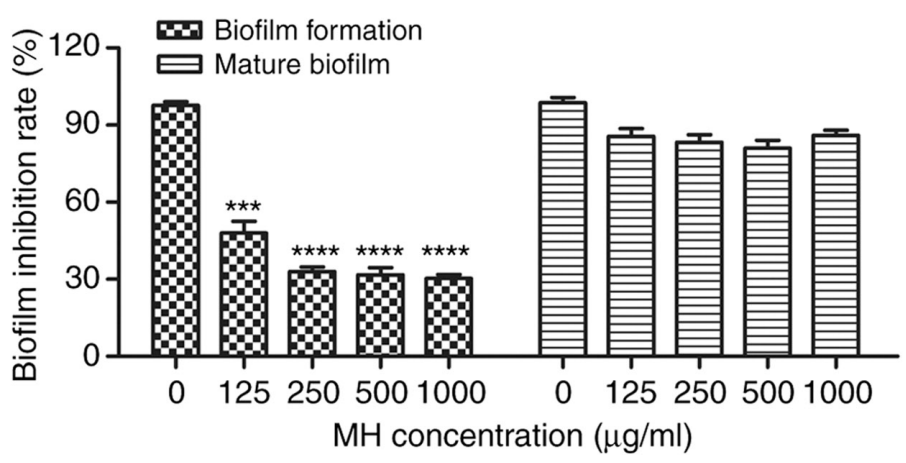

Figure 4. (A) Light microscopy images of biofilm formation and mature biofilm. With the increase in the MH concentration, the inhibition of biofilm formation was more obvious. At $250 \mu \mathrm{g} / \mathrm{ml} \mathrm{MH}$, there was a marked decrease in the density of biofilms. By contrast, in the presence of MH, mature biofilms exhibited no changes obvious changes observed by standard light microscopy (magnification, x1,000) (B) Effects of MH on biofilm formation and mature biofilms. MH inhibited $C$. albicans biofilm formation in vitro. Biofilm inhibition was evaluated using an XTT reduction assay and the results are presented as the percentage of $\mathrm{MH}$-treated biofilms relative to control biofilms that were formed without drug treatment. ${ }^{* * * *} \mathrm{P}<0.0001$ and ${ }^{* * * *} \mathrm{P}<0.001$ vs. control. MH, minocycline; C. albicans, Candida albicans. 


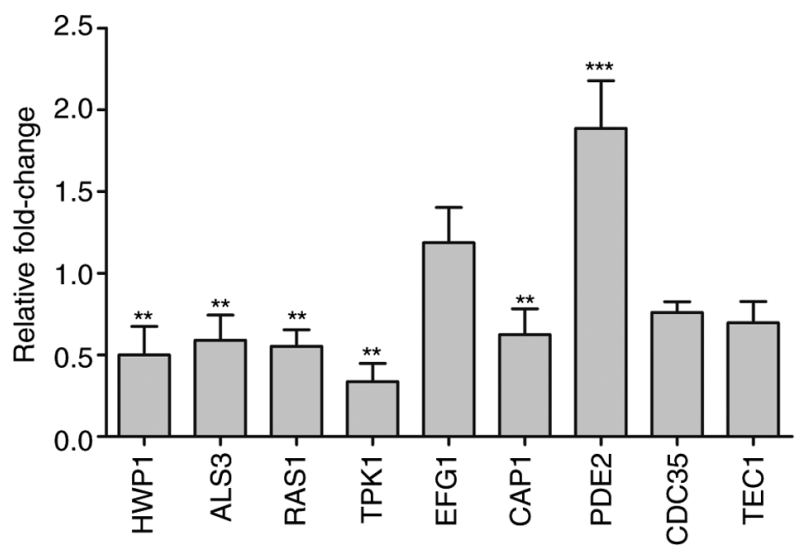

Figure 5. Gene expression levels in the C. albicans strain ATCC 90028. Fold change values of the expression of each target gene are shown relative to the values of the control group containing untreated $C$. albicans. The housekeeping gene ACT1 was used as an internal reference gene. The expression level of each target gene in the $\mathrm{MH}$-free sample was set as $1{ }^{* *} \mathrm{P}<0.01$; ${ }^{* * * *} \mathrm{P}<0.001$. C. albicans, Candida albicans; $\mathrm{MH}$, minocycline.

vs. control), and with the increase of the MH concentration, the inhibitory effect became more pronounced. $\mathrm{MH}$ inhibited biofilm formation by $67 \%$ at $250 \mu \mathrm{g} / \mathrm{ml}$ ( $\mathrm{P}<0.001$ vs. control). However, $\mathrm{MH}$ had no marked activity against the mature biofilms, which exhibited no obvious/significant changes following treatment with any of the $\mathrm{MH}$ concentrations. In conclusion, the tendency of $\mathrm{MH}$ to inhibit biofilm formation was confirmed.

$M H$ regulates the expression of $c A M P$ pathway genes. The expression levels of 9 genes were monitored by RT-qPCR. Hypha-specific genes from the adhesion process, $H W P 1$ and $A L S 3$, which are downstream components of the cAMP/PKA pathway, are positively regulated by EFG1 (25), and they were downregulated by 0.48 - and 0.59 -fold, respectively, following $250 \mu \mathrm{g} / \mathrm{ml} \mathrm{MH}$ treatment. Certain hyphal transcriptional regulation genes from the cAMP/PKA pathway, including $R A S 1, T P K 1, E F G 1, C A P 1, P D E 2$ and $C D C 35$, were also examined (Fig. 5). The upregulated genes included EFG1 and PDE2. The expression levels of EFG1 were slightly, though not significantly upregulated by $\mathrm{MH}$ treatment in the present study, while the upstream components $R A S 1, T P K 1$ and $C A P 1$ were downregulated by $0.55-$, 0.33 - and 0.62-fold, respectively. CDC35 and TEC1 were downregulated by 0.76 - and 0.69 -fold, respectively. Taken together, the results suggested that genes downregulated following $\mathrm{MH}$ treatment, including $H W P 1, A L S 3$, RAS1 and $T P K 1$, were all associated with the AMP pathway and regulated by the Ras/cAMP pathway (16).

\section{Discussion}

The results of the present study demonstrated that the antifungal activity of $\mathrm{MH}$ was mediated by regulatory factors in the Ras/cAMP pathway, which was conducive to the reduction of biofilm formation and limiting of hyphae formation and long-term maintenance of C. albicans.

It was indicated that MH had different inhibitory effects on different strains of fungus. It had a strong antifungal activity on yeasts such as $C$. albicans, $C$. tropicalis and $C$.lusitaniae, with the inhibition zones being $>14 \mathrm{~mm}$ and demonstrating a significant activity against $C$. albicans. $\mathrm{MH}$ had no inhibitory activity against $C$. pseudosmooth, $C$. glabrata and C. parapsilosis, while TE, DO and TGC had no significant antifungal properties. The antifungal effect of $\mathrm{MH}$ was marked as compared to other TE derivative agents. This may be linked to the morphological transformation and virulence in fungi (26); further research is required in the future to confirm this.

In addition, several studies have verified that the length and thickness during the growth of filamentous fungi directly affects the pathogenicity of hyphae, with the two being positively correlated $(27,28)$. The yeast form of $C$. albicans is less pathogenic and has a better adhesion capacity than the hyphae form due to its small and single form, at least in part because the yeast form is more easily eliminated by the defense system of the host than the hyphae form (29). In Spider and RPMI-1640 media, nutrient-rich conditions were added with $20 \%$ FBS in order for $C$. albicans to switch to the hyphae form more rapidly. However, in the present study, $\mathrm{MH}$ was able to maintain the yeast form for longer and inhibited the formation of hyphae, while MH clearly affected the hypha growth of $C$. albicans at the concentrations of 125 and $250 \mu \mathrm{g} / \mathrm{ml}$. In RPMI-1640 medium and Spider medium, the hypha rate (\%) of $125 \mathrm{MH}$ treatment was the most significant, $11.00 \pm 3.61$ and $9.67 \pm 2.51$, respectively. These results are consistent with those by Shi et al (10), who reported that when $\mathrm{MH}$ was combined with fluconazole, the minimum inhibitory concentration on C.albicans was decreased as compared with that of fluconazole used alone. Although there have been numerous reports on the phenomenon of antifungal agents used alone or combined with antimicrobial agents through $C$. albicans biofilms (13-15), few have investigated the particular antifungal characteristics of antimicrobial agents in $C$. albicans. To explore the potential mechanisms, the interference ability of $\mathrm{MH}$ to penetrate biofilms was first assessed.

C. albicans biofilms have a complex and intricate three-dimensional network architecture, with the barrier characteristics of $C$. albicans biofilms mainly reflected in an increased tolerance to traditional antifungal therapy. An XTT reduction assay was performed to demonstrate the suppressive function of $\mathrm{MH}$ on biofilms, and it was indicated that $\mathrm{MH}$ had a distinct inhibitory effect against $C$. albicans biofilms. The results suggested that $250 \mu \mathrm{g} / \mathrm{ml} \mathrm{MH}$ significantly depressed biofilm formation by $67 \%(\mathrm{P}<0.001)$. With the increase in concentration, the inhibitory activity of $\mathrm{MH}$ tended to be stable and changes showed no significant difference, indicating that the optimal inhibitory effect had been achieved or approached at $250 \mu \mathrm{g} / \mathrm{ml}$. Of note, it exhibited no activity to impair the maintenance of mature biofilms. In conclusion, $\mathrm{MH}$ had a strong antibiofilm effect against $C$. albicans in vitro, which appears to be attributable to its anti-morphological transition activities.

According to the inhibition of $C$. albicans filamentous growth and biofilm formation, the transcription levels of genes associated with mycelium growth, adhesion and biofilm formation in $C$. albicans were determined to further clarify the molecular mechanism of the anti-biofilm effects of $\mathrm{MH}$. The analysis revealed that several important genes were differentially expressed following $\mathrm{MH}$ treatment. CAPI, 
TPK1, CDC35, TECI, RAS1, ALS3 and HWP1 were downregulated, and $E F G 1$ and $P D E 2$ were upregulated following treatment with $250 \mu \mathrm{g} / \mathrm{ml} \mathrm{MH}$. In this assay, MH was applied at $250 \mu \mathrm{g} / \mathrm{ml}$, since at this concentration had a significant antibiofilm activity. It should be noted that these downregulated genes are all important components of the RAS1/cAMP/PKA signaling pathway; the present hypothesis that a direct interaction between the pathways occurs at the transcriptional level was confirmed. Previous studies have reported that several signaling pathways are linked to morphogenesis and biofilms in C.albicans, with the MAPK and cAMP/PKA signaling pathways being the most prominent $(16,30,31)$. The cAMP/PKA pathway has attracted extensive attention in fungal pathogens such as C. albicans, Trichophyton, Cryptococcus neoformans and Aspergillus fumigatus $(32,33)$, due to its vital function in the specific morphology development of fungal biofilms. The Ras/AMP/PKA pathway is involved in the regulation of various traits of $C$. albicans and responds to specific environmental stimuli and cell subtype combinations (30).

In the present study, the most significantly downregulated genes were RAS1 and TPK1 (0.55- and 0.33-fold, respectively). RAS1 has a vital role in the morphological switch and biofilm formation of yeast and hypha $(34,35)$. It is a soluble small GTPase that transmits signals to stimulate filamentous growth in the Ras1/cAMP/PKA signaling pathway as a transcription factor (36-38). Despite RAS1 gene mutation and deletion of alleles, which resulted in serious hyphal growth defects, such as deformation and irregularity in response to serum, and inhibition of pseudohyphal development (34), studies have indicated that the addition of sufficient exogenous cAMP or overexpressed components of MAP kinase cascade in the growth media is able to correct the above morphological defects and make mycelium return to normal $(35,39)$. Evidence suggested that the RAS1 gene is an intermediate factor in the anti-fungal regulation of $\mathrm{MH}$, which is involved in signaling pathway regulation through the RASl gene to interfere with biofilms (14).

Recent research has demonstrated that the deletion of TPK1 results in hyphal formation defects on solid media in C. tropicalis (40). In GlcNAc-inducing liquid medium, TPKI was associated with the appearance of germ-tube, with the mRNA levels of TPK1 increasing gradually with cell growth and peaking at the onset of germ-tube emergence (41). Lin and Chen (40) reported that PDE2 tightly regulated the intracellular cAMP level. Deficiency of PDE2 led to the activation of cAMP, which decreased the thickness of the cell wall and cell membrane of the fungus, therefore reducing the virulence of the fungus and making cells more sensitive to antifungal agents. High expression of PDE2 inhibited cAMP synthesis and limited hypha production (42), while all the defects observed in hyphal morphogenesis in vitro were reversible and rectifiable. The present results suggested that the increase in PDE2 mRNA following MH intervention was most significant, which was consistent with the results of previous study (43), which further explained the inhibition of PDE2 on hypha and biofilm growth in C. albicans.

EFG1 is a well-characterized transcription factor and important component of the Ras/cAMP pathway, which may both bidirectionally regulate genes that participate in the essential processes and stages of cellular growth, such as white-opaque transition in morphogenesis, and glycolysis and respiration in cell metabolism $(44,45)$. Furthermore, EFG1 acts upstream of TEC1 and is located in the middle stream of the RAS1/cAMP-PKA signaling pathway, which regulates and controls the upstream and downstream components, including HWP1, TCE1 and ALS3 (42). Mutational analysis suggested that deficiency of the EFG1 gene disrupted normal morphological transformation, impeded the growth of mycelium, induced hyphae in a pseudorevertant strain and was dependent on the EFG1 transcription factor that has a regulatory role in the downstream of protein kinase A (46). TEC1, located downstream of EFG1, also regulates the growth of hyphae-specific genes, with the EFG1/efg1 mutants resulting in the overexpression of TEC1 (47). Deletion of TEC1 resulted in thinner biofilms and the observed disordered growth of short spores on their surface. In the present study, a slight elevation of EFG1 was observed and the modulating effect of MH on EFG1 was not significant. In the present results, most aberrant genes were downregulated genes, which were all important components of the RAS1/cAMP/PKA signaling pathway, while fewer genes were upregulated. These results suggested that the signaling pathways of MH may be dominated by negative regulation and that the RAS1/cAMP/PKA signaling pathway may have a crucial role in the regulation of hypha and biofilm by MH.

However, the present study had certain limitations. For instance, due to the limited resources and funding available, no resistant strain analysis was performed and the effects of MH on drug-resistant strains of C. albicans were not analyzed. In addition, animal studies are required to verify the drug toxicity and feasible doses of MH. Further experiments, such as western blot analysis to detect changes in the protein levels, may be performed, or changes in electrolytes in the biofilm, such as ionic calcium, may be assessed in order to investigate the mechanism of action of MH. Validation using clinical samples is also required. However, despite these limitations, the results of the present study may provide novel insight that may lead to the development of clinical medication and treatment.

In conclusion, the present study demonstrated that $\mathrm{MH}$ exhibits a strong anti-biofilm effect against $C$. albicans selectively, and the predominant mechanisms are associated with the Ras/cAMP pathway. Therefore, the hypothesis of the present study, that the antifungal effect of $\mathrm{MH}$ on C.albicans is mainly associated with biofilm formation and its regulation is closely associated with the RAS/cAMP/PKA pathway was validated. These results indicated that MH may be an effective antifungal agent for $C$. albicans infections. However, clinical application is not yet possible, as more prospective studies using strains and animal models are required, and treatment strategies for drug-resistant $C$. albicans infection require to be developed to further verify the clinical applicability of $\mathrm{MH}$. This is the direction of our future research.

\section{Acknowledgements}

Not applicable.

\section{Funding}

This work was supported by grants from the Health Committee Union Foundation of Hubei Province (grant no. WJ2019H512) 
and the Youth Science Foundation of Three Gorges University (grant no. KJ2018A007).

\section{Availability of data and materials}

The datasets used and/or analyzed during the current study are available from the corresponding author on reasonable request.

\section{Authors' contributions}

LZ and QD designed the experiments and drafted the manuscript. ZM performed the experiments and analyzed the data. TG and BZ performed the RT-qPCR experiments. QD reviewed the manuscript. All authors read and approved the final manuscript.

\section{Ethics approval and consent to participate}

Not applicable.

\section{Patient consent for publication}

Not applicable.

\section{Competing interests}

The authors declare that they have no competing interests.

\section{References}

1. Gow NA, van de Veerdonk FL, Brown AJ and Netea MG: Candida albicans morphogenesis and host defence: Discriminating invasion from colonization. Nat Rev Microbiol 10: 112-122, 2011

2. Ferreira AV, Prado CG, Carvalho RR, Dias KS and Dias AL: Candida albicans and non- $C$. albicans Candida species: Comparison of biofilm production and metabolic activity in biofilms, and putative virulence properties ofisolates from hospital environments and infections. Mycopathologia 175 265-272, 2013

3. Chandra J, Mukherjee PK, Leidich, SD, Faddoul FF, Hoyer LL, Douglas LJ and Ghannoum MA: Antifungal resistance of candidal biofilms formed on denture acrylic in vitro. J Dent Res 80: 903-908, 2001.

4. Karagoz E, Ugan RA, Duzgun E, Cadirci E, Keles S, Uyanik MH, Yavan I and Turhan V: Comparative study of the effects of intravitreal anidulafungin, voriconazole, and amphotericin B in an experimental Candida endophthalmitis model. Curr Eye Res 42 : 225-232, 2017.

5. Pfaller MA, Rhomberg PR, Messer SA, Jones RN and Castanheira M: Isavuconazole, micafungin, and 8 comparator antifungal agents' susceptibility profiles for common and uncommon opportunistic fungi collected in 2013: Temporal analysis of antifungal drug resistance using CLSI species-specific clinical breakpoints and proposed epidemiological cutoff values. Diagn Microbiol Infect Dis 82: 303-313, 2015.

6. Noble SM, Gianetti BA and Witchley JN: Candida albicans cell-type switching and functional plasticity in the mammalian host. Nat Rev Microbiol 15: 96-108, 2017.

7. Sudbery PE: Growth of Candida hyphae. Nat Rev Microbiol 9: 737-748, 2011

8. Li DD, Xu Y, Zhang DZ, Quan H, Mylonakis E, Hu DD, Li MB Zhao LX, Zhu LH, Wang Y and Jiang YY: Fluconazole assists berberine to kill fluconazole-resistant Candida albicans. Antimicrob Agents Chemother 57: 6016-6027, 2013

9. Nett JE, Sanchez H, Cain MT, Ross KM and Andes DR: Interface of Candida albicans biofilm matrix-associated drug resistance and cell wall integrity regulation. Eukaryot Cell 10: 1660-1669, 2011.
10. Shi W, Chen ZZ, Chen X, Cao L, Liu P and Sun S: The combination of minocycline and fluconazole causes synergistic growth inhibition against Candida albicans: An in vitro interaction of antifungal and antibacterial agents. FEMS Yeast Res 10: 885-893, 2010.

11. de Oliveira LF, Jorge AC and Santos SF: In vitro minocycline activity on superinfecting microorganisms isolated from chronic periodontitis patients. Braz Oral Res 20: 202-206, 2006.

12. Raad I, Darouiche R, Hachem R, Sacilowski M and Bodey GP: Antibiotics and prevention ofmicrobial colonization of catheters. Antimicrob Agents Chemother 39: 2397-2400, 1995.

13. Gao Y, Li H, Liu S, Zhang X and Sun S: Synergistic effect of fluconazole and doxycycline against Candida albicans biofilms resulting from calcium fluctuation and downregulation of fluconazole-inducible efflux pump gene overexpression. J Med Microbiol 63: 956-961, 2014.

14. Kurakado S, Takatori K and Sugita T: Minocycline inhibits Candida albicans budded-to-hyphal-form transition and biofilm formation. Jpn J Infect Dis 70: 490-494, 2017.

15. Li H, Liu P, Liu W, Gao Y and Sun S: In vitro interactions between fluconazole and minocycline against mixed cultures of Candida albicans and Staphylococcus aureus. J Microbiol Immunol Infect 48: 655-661, 2015.

16. Hogan DA and Sundstrom P: The Ras/cAMP/PKA signaling pathway and virulence in Candida albicans. Future Microbiol 4: 1263-1270, 2009.

17. Phillips AJ, Crowe JD and Ramsdale M: Ras pathway signaling accelerates programmed cell death in the pathogenic fungus Candida albicans. Proc Natl Acad Sci USA 103: 726-731, 2006.

18. Li DD, Zhao LX, Mylonakis E, Hu GH, Zou Y, Huang TK, Yan L, Wang Y and Jiang YY: In vitro and in vivo activities of pterostilbene against Candida albicans biofilms. Antimicrob Agents Chemother 58: 2344-2355, 2014.

19. Gimeno CJ, Ljungdahl PO, Styles CA and Fink GR: Unipolar cell divisions in the yeast $\mathrm{S}$. cerevisiae lead to filamentous growth: Regulation by starvation and RAS. Cell 68: 1077-1090, 1992.

20. Zhong H, Hu DD, Hu GH, Su J, Bi S, Zhang ZE, Wang Z, Zhang RL, Xu Z, Jiang YY and Wang Y: Activity of sanguinarine against Candida albicans biofilms. Antimicrob Agents Chemother 61: 02259-16, 2017.

21. Ma C, Du F, Yan L, He G, He J, Wang C, Rao G, Jiang Y and $\mathrm{Xu}$ G: Potent activities of roemerine against Candida albicans and the underlying mechanisms. Molecules 20: 17913-17928, 2015.

22. Ku TS, Palanisamy S K and Lee SA: Susceptibility of Candida albicans biofilms to azithromycin, tigecycline and vancomycin and the interaction between tigecycline and antifungals. Int J Antimicrob Agents 36: 441-446, 2010.

23. Cao S, Zhang X, Ye N, Fan X, Mou S, Xu D, Liang C, Wang Y and Wang W: Evaluation of putative internal reference genes for gene expression normalization in Nannochloropsis sp. by quantitative real-time RT-PCR. Biochem Biophys Res Commun 424: 118-123, 2012.

24. Livak KJ and Schmittgen TD: Analysis of relative gene expression data using real-time quantitative PCR and the 2(-Delta Delta C(T)) method. Methods 25: 402-408, 2001.

25. Langford ML, Hargarten JC, Patefield KD, Marta E, Blankenship JR, Fanning S, Nickerson KW and Atkina AL: Candida albicans $\mathrm{Czf1}$ and Efg1 coordinate the response to farnesol during quorum sensing, white-opaque thermal dimorphism, and cell death. Eukaryot Cell 12: 1281-1292, 2013.

26. Ramírez-Zavala B, Weyler M, Gildor T, Schmauch C, Kornitzer D, Arkowitz R and Morschhäuser J: Activation of the Cph1-dependent MAP kinase signaling pathway induces white-opaque switching in Candida albicans. PLoS Pathog 9: e1003696, 2013.

27. Berman J and Sudbery PE: Candida albicans: A molecular revolution built on lessons from budding yeast. Nat Rev Genet 3: 918-930, 2002.

28. Biswas S, Dijck PV and Datta A: Environmental sensing and signal transduction pathways regulating morphopathogenic determinants of Candida albicans. Microbiol Mol Biol Rev 71: 348-376, 2007.

29. Huh WK and Kang SO: Molecular cloning and functional expression of alternative oxidase from Candida albicans. J Bacteriol 181: 4098-4102, 1999.

30. Huang G, Huang Q, Wei Y, Wang Y and Du H: Multiple roles and diverse regulation of the Ras/cAMP/protein kinase A pathway in Candida albicans. Mol Microbiol 111: 6-16, 2019. 
31. Stoldt VR, Sonneborn A, Leuker CE and Ernst JF: Efg1p, an essential regulator of morphogenesis of the human pathogen Candida albicans, is a member of a conserved class of bHLH proteins regulating morphogenetic processes in fungi. EMBO J 16: 1982-1991, 1997.

32. Fuller KK and Rhodes JC: Protein kinase A and fungal virulence: A sinister side to a conserved nutrient sensing pathway. Virulence 3: 109-121, 2012.

33. Cloutier M, Castilla R, Bolduc N, Zelada A, Martineau P, Bouillon M, Magee BB, Passeron S, Giasson L and Cantoreb ML: The two isoforms of the cAMP-dependent protein kinase catalytic subunit are involved in the control of dimorphism in the human fungal pathogen Candida albicans. Fungal Genet Biol 38: 133-141, 2003.

34. Feng Q, Summers E, Guo B and Fink G: Ras signaling is required for serum-induced hyphal differentiation in Candida albicans. J Bacteriol 181: 6339-6346, 1999.

35. Leberer E, Harcus D, Dignard D, Johnson L, Ushinsky S, Thomas DY and Schröppel K: Ras links cellular morphogenesis to virulence by regulation of the MAP kinase and CAMP signalling pathways in the pathogenic fungus Candida albicans. Mol microbiol 42: 673-687, 2001.

36. Cullen PJ and Sprague GJ Jr: The regulation of filamentous growth in yeast. Genetics 190: 23-49, 2012.

37. Lu Y, Su C and Liu H: Candida albicans hyphal initiation and elongation. Trends Microbiol 22: 707-714, 2014.

38. Davis-Hanna A,Piispanen AE,StatevaLI and Hogan DA: Farnesol and dodecanol effects on the Candida albicans Ras1-cAMP signalling pathway and the regulation of morphogenesis. Mol Microbiol 67: 47-62, 2008.

39. Zhu Y, Fang HM, Wang YM, Zeng GS, Zheng XD and Wang Y: Ras1 and Ras2 play antagonistic roles in regulating cellular cAMP level, stationary-phase entry and stress response in Candida albicans. Mol Microbiol 74: 862-875, 2009.

40. Lin CJ, Wu CY, Yu SJ and Chen YL: Protein kinase A governs growth and virulence in Candida tropicalis. Virulence 9: 331-347, 2018.
41. Souto G, Giacometti R, Silberstein S, Giasson L, Cantore ML and Passeron S: Expression of TPK1 and TPK2 genes encoding PKA catalytic subunits during growth and morphogenesis in Candida albicans. Yeast 23: 591-603, 2006.

42. Lin CJ and Chen YL: Conserved and divergent functions of the cAMP/PKA signaling pathway in Candida albicans and Candida tropicalis. J Fungi (Basel) 4: 68, 2018.

43. Bahn YS, Staab J and Sundstrom P: Increased high-affinity phosphodiesterase PDE2 gene expression in germ tubes counteracts CAP1-dependent synthesis of cyclic AMP, limits hypha production and promotes virulence of Candida albicans. Mol Microbiol 50: 391-409, 2003.

44. Nobile CJ, Fox EP, Nett JE, Sorrells TR, Mitrovich QM, Hernday AD, Tuch BB, Andes DR and Johnson AD: A recently evolved transcriptional network controls biofilm development in Candida albicans. Cell 148: 126-138, 2012.

45. Tebarth B, Doedt T, Krishnamurthy S, Weide M, Monterola F, Dominguez A and Ernst JF: Adaptation of the Efglp morphogenetic pathway in Candida albicans by negative autoregulation and PKA-dependent repression of the EFG1 gene. J Mol Biol 329: 949-962, 2003.

46. Parrino SM, Si H, Naseem S, Groudan K, Gardin J and Konopka JB: cAMP-independent signal pathways stimulate hyphal morphogenesis in Candida albicans. Mol Microbiol 103: 764-779, 2017.

47. Lopes da Rosa J and Kaufman PD: Chromatin-mediated Candida albicans virulence. Biochim Biophys Acta 1819: 349-355, 2012.

c) (i) $\odot$ This work is licensed under a Creative Commons cc) Attribution-NonCommercial-NoDerivatives 4.0 International (CC BY-NC-ND 4.0) License. 